\title{
Role of Age, Sex, and Race on Cardiac and Total Mortality Associated With Super Bowl Wins and Losses
}

\author{
Robert A. Kloner, MD, PhD; Scott A. McDonald, BS; Justin Leeka; W. Kenneth \\ Poole, PhD \\ Heart Institute (Kloner, Leeka), Good Samaritan Hospital, Los Angeles, California; Keck School of \\ Medicine (Kloner), University of Southern California, Los Angeles, California; RTI International \\ (McDonald, Poole), Research Triangle Park, North Carolina
}

\begin{abstract}
Background: Total and cardiac mortality rates in Los Angeles County, Califorina, increased after the 1980 Super Bowl loss (SBL), but there was an overall reduction in total mortality after the 1984 Super Bowl win (SBW). Hypothesis: We hypothesized that age, sex, and race may have played a role in the Super Bowl related differences in death rates.

Methods: We compared mortality rates for SB-related days with non-SB control days assessing differences in demographics. We ran regression models predicting daily death rates per 100,000 including SB variable versus non-SB control days for age, sex, race, and interactions for these covariates.

Results: After the SBL, daily death rates increased for both males and females. People aged $\geq 65$ years had a larger absolute increase in all cause mortality during the SBL days compared with those aged $<65$ years, with significant interaction between age and SBL-variable for all-cause and cardiac-related mortality. Whites and Hispanics had increased death rates on SBL days. There were trends suggesting less death in older patients and females associated with the SBW.

Conclusion: A SBL triggered increased deaths in both men and women and especially in older patients, whereas a SBW reduced death more in those aged $\geq 65$ years and in women.
\end{abstract}

\section{Introduction}

There are both chronic risk factors for the development of heart disease as well as acute risk factors that may trigger an acute coronary event. Chronic risk factors associated with the development of atherosclerosis include dyslipidemia, diabetes, smoking, hypertension, metabolic syndrome, lack of physical exertion, and obesity. ${ }^{1}$ Acute risk factors usually involve some form of stress-physical, emotional, or both - that increases the sympathetic nervous system and releases catecholamines. ${ }^{2-7}$ The subsequent increase in heart rate, blood pressure, and ventricular contractility increases oxygen demand and may change the shear stress of blood against an atherosclerotic plaque, contributing to plaque fracture. Stimulation of alpha receptors in the vasculature may actually increase vascular resistance, increasing afterload and constricting coronary vessels (including frank coronary vasospasm), thus increasing oxygen demand at the same time as limiting oxygen supply to the heart. Catecholamine excess can also increase platelet aggregability as well as contribute to arrhythmias.

The authors have no funding, financial relationships, or conflicts of interest to disclose.
Situations in which certain stressors can trigger cardiovascular events, thought to occur through these mechanisms, include emotional stress, heavy physical exertion, moderate physical exertion, lack of sleep, overeating, and a host of other stressful situations. ${ }^{5}$ Sports fans may demonstrate an increase in cardiac events during emotionally charged soccer games, as demonstrated from analyses during World Cup soccer. ${ }^{8}$ Previously, it was observed that total and cardiac mortality rates in Los Angeles County were increased during the 1980 Super Bowl loss; in contrast, there was an overall reduction in total mortality associated with the 1984 Super Bowl win. ${ }^{9}$ To what extent age, sex, or race played a role in these deaths is unknown. Therefore, we analyzed data on men, women, those aged $<65$ years, those aged $\geq 65$ years, those of white/Hispanic ethnicity, and those of nonwhite/non-Hispanic ethnicity, considering the effect of both a Super Bowl win and a Super Bowl loss on total as well as cardiac mortality in Los Angeles County.

\section{Background}

Los Angeles has played twice in the Super Bowl. The first time was the Los Angeles Rams vs the Pittsburgh Steelers, which was played locally (at the Rose Bowl, Pasadena, California) on January 20, 1980. The Rams had been in Los Angeles for many years, were a much-loved team, and this 
game was high intensity, with seven lead changes before Los Angeles lost a fourth-quarter lead and the game. In contrast, Los Angeles's only winning Super Bowl was played on January 22, 1984, between the Los Angeles Raiders and the Washington Redskins. At the time, the Raiders had only been in Los Angeles for a few years. Los Angeles carried the lead from the beginning, and this was a less-intense game. In addition, this winning game was played outside of Los Angeles County, in Tampa, Florida.

\section{Methods}

We obtained death-certificate data from the Department of Health Sciences in Los Angeles County from 1980 to 1988 , covering only the period of January 15 through the end of February for each year. Data included total number of deaths as well as number of deaths due to diseases of the circulatory system, ischemic heart disease, acute myocardial infarction, heart failure, and congestive heart failure. Data on the entire population have been previously published. ${ }^{9}$ In the present study, we separately analyzed data on men, women, those aged $<65$ years, those aged $\geq 65$ years, those of white/Hispanic ethnicity, and those of nonwhite/non-Hispanic ethnicity, for each of the 2 Super Bowls. We compared death rates for days related to the 1980 Super Bowl (January 20, 1980 plus the following 14 days), which Los Angeles lost, with control days (all other days in January and February from January 15, 1980 to February 1983). To remove the impact of the known peak in total and cardiac death rates around the winter holidays, all analyses excluded data from January 1 to January 14 . Death rates were reported as deaths per day per 100000 population (using total deaths and average population). Parallel analyses were performed for Los Angeles's winning Super Bowl on January 22, 1984. During these time periods, Los Angeles County death certificates classified Hispanic patients as white; hence, white and Hispanic death-rate data are combined.

One-way analysis of variance was used to compare mortality rates for the Super Bowl-related days with control days, and $P$ values for the Super Bowl effect were considered statistically significant at $<0.05$. In addition, we ran a linear regression model predicting death rate, including the following variables in the model: the Super Bowl variable (day of Super Bowl and subsequent 14 days vs the other days), race (white/Hispanic vs nonwhite, non-Hispanic), sex (male vs female), age ( $<65$ years vs $\geq 65$ years), plus interactions for each of these covariates with the Super Bowl variable.

\section{Results}

\section{Los Angeles's 1980 Super Bowl Loss}

Analysis of all-cause and cardiac mortality rates for the 1980 Super Bowl and subsequent 14 days vs all other days in January and February between January 15, 1980, and February 1983 are shown for males and females in Table 1. In men, mortality rate due to any cause was higher during the losing Super Bowl days than on control days. In women, mortality rate due to any cause was also higher during the 1980 losing Super Bowl days compared with control days. Circulatory deaths were higher during Super Bowl days in both men and women.
Table 1. Analysis of Sex and Deaths From Cardiac Causes for the 1980 Super Bowl and Subsequent 14 Days $^{a}$ vs All Other Days in January ${ }^{b}$ and February Between January 15, 1980, and February 1983

\begin{tabular}{|c|c|c|c|c|}
\hline Cause of Death & Control $^{C}$ & Super Bowl $1980^{C}$ & $\beta$ Estimate $^{d}$ & $P$ Value $^{e}$ \\
\hline \multicolumn{5}{|l|}{ Male } \\
\hline All circ. & 1.04 & 1.20 & 0.16 & 0.003 \\
\hline IHD & 0.74 & 0.85 & 0.12 & 0.01 \\
\hline AMI & 0.25 & 0.29 & 0.04 & 0.11 \\
\hline $\mathrm{HF} / \mathrm{CHF}$ & 0.014 & 0.013 & -0.001 & 0.86 \\
\hline All deaths & 2.20 & 2.50 & 0.29 & 0.001 \\
\hline \multicolumn{5}{|l|}{ Female } \\
\hline All circ. & 1.10 & 1.40 & 0.30 & $<0.0001$ \\
\hline IHD & 0.69 & 0.86 & 0.16 & 0.0002 \\
\hline AMI & 0.22 & 0.26 & 0.04 & 0.06 \\
\hline $\mathrm{HF} / \mathrm{CHF}$ & 0.017 & 0.014 & -0.003 & 0.56 \\
\hline All deaths & 1.99 & 2.40 & 0.41 & $<0.0001$ \\
\hline
\end{tabular}

Abbreviations: AMI, acute myocardial infarction; ANOVA, analysis of variance; Circ., diseases of the circulatory system; HF/CHF, heart failure or congestive heart failure; IHD, ischemic heart disease.

${ }^{a}$ The analysis group thus includes the day of the Super Bowl (January 20, 1980), and the 14 days after the Super Bowl (January 20, 1980-February 3, 1980, 15 days total).

${ }^{b}$ To remove the impact of the known peak in cardiac death rates around the holidays, the analysis excludes data from January 1 to 14 (14 days starting with New Year's Day).

${ }^{C}$ Rates expressed per day per 100000 population (using total deaths and average population).

${ }^{d}$ Estimated from a 1-way ANOVA with Super Bowl Day as the only factor.

${ }^{e}$ For the Super Bowl effect.

Mortality rates for those aged $<65$ years were higher on Super Bowl days than on control days $(P=0.004$; Table 2$)$. For those aged $\geq 65$ years (Table 2 ), total mortality rates were higher, as expected, and there was a highly significant $(P<0.0001)$ increase in total mortality rates per day associated with the losing Super Bowl days (an absolute increase of 2.6 deaths per day for those aged $\geq 65$ and 0.11 for those aged $<65)$. There was also a highly significant worsening of all circulatory deaths $(P<0.0001)$ and ischemic heart deaths $(P=0.0001)$ in those aged $\geq 65$ years.

Mortality rates for white/Hispanic and nonwhite/nonHispanic populations are shown in Table 3 . There were significant increases in all-cause death rates, death from heart disease, and death from ischemic heart disease associated with the 1980 losing Super Bowl days; there were trends in the same direction for nonwhite/non-Hispanic persons, but these were not statistically significant.

Table 4 shows linear regression analyses for death rate for the 1980 Super Bowl data with the following variables included in the model: Super Bowl (day of Super Bowl and subsequent 14 days vs other days), race (white and Hispanic vs nonwhite/non-Hispanic), sex (male vs female), age $(<65$ years vs $\geq 65)$, and interaction for each of these covariates with the Super Bowl variable (last 3 columns of 
Table 2. Analysis of Age and Deaths From Cardiac Causes for the 1980 Super Bowl and Subsequent 14 Days $^{a}$ vs All Other Days in January ${ }^{b}$ and February Between January 15, 1980, and February 1983

\begin{tabular}{|c|c|c|c|c|}
\hline Cause of Death & Control $^{c}$ & Super Bowl $1980^{C}$ & $\beta$ Estimate $^{d}$ & $P$ Value $e^{e}$ \\
\hline \multicolumn{5}{|l|}{ Age $<65$ y } \\
\hline All circ. & 0.23 & 0.28 & 0.05 & 0.002 \\
\hline IHD & 0.15 & 0.18 & 0.03 & 0.01 \\
\hline AMI & 0.05 & 0.07 & 0.01 & 0.12 \\
\hline $\mathrm{HF} / \mathrm{CHF}$ & 0.002 & 0.001 & -0.001 & 0.47 \\
\hline All deaths & 0.79 & 0.90 & 0.11 & 0.004 \\
\hline \multicolumn{5}{|l|}{ Age $\geq 65 y$} \\
\hline All circ. & 8.76 & 10.7 & 1.90 & $<0.0001$ \\
\hline IHD & $5 \cdot 91$ & 7.02 & 1.10 & 0.0001 \\
\hline AMI & 1.87 & 2.15 & 0.27 & 0.06 \\
\hline $\mathrm{HF} / \mathrm{CHF}$ & 0.139 & 0.127 & -0.01 & 0.76 \\
\hline All deaths & 14.1 & 16.7 & 2.57 & $<0.0001$ \\
\hline
\end{tabular}

Abbreviations: AMI, acute myocardial infarction; ANOVA, analysis of variance; Circ., diseases of the circulatory system; HF/CHF, heart failure or congestive heart failure; IHD, ischemic heart disease.

${ }^{a}$ The analysis group thus includes the day of the Super Bowl (January 20, 1980), and the 14 days after the Super Bowl (January 20, 1980-February 3, 1980, 15 days total).

${ }^{b}$ To remove the impact of the known peak in cardiac death rates around the holidays, the analysis excludes data from January 1 to 14 (14 days starting with New Year's Day).

${ }^{c}$ Rates expressed per day per 100000 population (using total deaths and average population).

${ }^{d}$ Estimated from a 1-way ANOVA with Super Bowl Day as the only factor.

${ }^{e}$ For the Super Bowl effect.

Table 4). Note that there was significant interaction between age and the Super Bowl variable, but not between race and Super Bowl or sex and Super Bowl. Hence, the Super Bowl loss triggered more deaths in older patients than in younger patients. The analyses use data from January 15 to February, 1980-1983. There are 181 days included in this interval, and there are 8 combinations of these covariates $(2 \times$ race, $2 \times$ sex, $2 \times$ age), for a total of 1448 observations (independent death rates).
Table 3. Analysis of Race and Deaths From Cardiac Causes for the 1980 Super Bowl and Subsequent 14 Days $^{a}$ vs All Other Days in January ${ }^{b}$ and February Between January 15, 1980, and February 1983

\begin{tabular}{|c|c|c|c|c|}
\hline Cause of Death & Control $^{C}$ & $\begin{array}{l}\text { uper Bowl } \\
1980^{c}\end{array}$ & $\beta$ Estimate $^{d}$ & $P$ Value $^{e}$ \\
\hline \multicolumn{5}{|l|}{ White/Hispanic } \\
\hline All circ. & 1.15 & 1.42 & 0.27 & $<0.0001$ \\
\hline IHD & 0.79 & 0.95 & 0.16 & $<0.0001$ \\
\hline AMI & 0.26 & 0.30 & 0.04 & 0.02 \\
\hline $\mathrm{HF} / \mathrm{CHF}$ & 0.017 & 0.013 & -0.004 & 0.41 \\
\hline All deaths & 2.21 & 2.62 & 0.41 & $<0.0001$ \\
\hline \multicolumn{5}{|c|}{ Nonwhite/non-Hispanic } \\
\hline All circ. & 0.71 & 0.79 & 0.07 & 0.21 \\
\hline IHD & 0.41 & 0.44 & 0.03 & 0.46 \\
\hline AMI & 0.13 & 0.15 & 0.02 & 0.48 \\
\hline $\mathrm{HF} / \mathrm{CHF}$ & 0.009 & 0.014 & 0.005 & 0.49 \\
\hline All deaths & 1.64 & 1.70 & 0.06 & 0.54 \\
\hline
\end{tabular}

Abbreviations: AMI, acute myocardial infarction; ANOVA, analysis of variance; Circ., diseases of the circulatory system; $\mathrm{HF} / \mathrm{CHF}$, heart failure or congestive heart failure; IHD, ischemic heart disease.

${ }^{a}$ The analysis group thus includes the day of the Super Bowl January 20, 1980), and the 14 days after the Super Bowl (January 20, 1980-February 3, 1980, 15 days total).

${ }^{b}$ To remove the impact of the known peak in cardiac death rates around the holidays, the analysis excludes data from January 1 to 14 (14 days starting with New Year's Day).

${ }^{C}$ Rates expressed per day per 100000 population (using total deaths and average population).

${ }^{d}$ Estimated from a 1-way ANOVA with Super Bowl Day as the only factor.

${ }^{e}$ For the Super Bowl effect.

\section{Los Angeles's 1984 Super Bowl Win}

Analysis of all-cause and cardiac mortality rates for the 1984 Super Bowl and subsequent 14 days vs all other days in January and February between January 15, 1984, and February 1988 are shown for males and females in Table 5. For women, but not men, there was a reduction in all-cause death and circulatory deaths associated with the Super Bowl win.

Table 4. $P$ Values From Linear Regression for Mortality Rate for the 1980 Super Bowl ${ }^{a}$

\begin{tabular}{|c|c|c|c|c|c|c|c|}
\hline Outcome & $1980 \mathrm{SB}$ & Race & Sex & Age & SB Race & SB Sex & SB Age \\
\hline All-cause mortality & $<0.0001$ & $<0.0001$ & $<0.0001$ & $<0.0001$ & 0.67 & 0.58 & $<0.0001$ \\
\hline IHD & 0.007 & $<0.0001$ & $<0.0001$ & $<0.0001$ & 0.69 & 0.87 & 0.009 \\
\hline AMI & 0.07 & 0.009 & 0.02 & $<0.0001$ & 0.63 & 0.56 & 0.08 \\
\hline $\mathrm{HF} / \mathrm{CHF}$ & 0.48 & 0.46 & 0.92 & $<0.0001$ & 0.23 & 0.80 & 0.45 \\
\hline
\end{tabular}

Abbreviations: AMI, acute myocardial infarction; HF/CHF, heart failure or congestive heart failure; IHD, ischemic heart disease; SB, Super Bowl.

${ }^{a}$ Analyses are linear regressions for mortality rate with the following variables included in the model: SB (day of SB and subsequent 14 days vs other days), race (white/Hispanic vs all others), sex (male vs female), age ( $<65$ years vs $\geq 65$ years), and interactions for each of these covariates with the SB variable. 
Table 5. Analysis of Sex and Deaths From Cardiac Causes for the 1984 Super Bowl and Subsequent 14 Days $^{a}$ vs All Other Days in January ${ }^{b}$ and February Between January 15, 1984, and February 1988

\begin{tabular}{|lcccc|}
\hline Cause of Death & Control $^{c}$ & $\begin{array}{c}\text { Super Bowl } \\
1984^{c}\end{array}$ & $\beta$ Estimate ${ }^{d}$ & $P$ Value $^{e}$ \\
\hline Male & & & & \\
\hline All circ. & 1.04 & 1.08 & 0.04 & 0.38 \\
\hline IHD & 0.73 & 0.76 & 0.03 & 0.51 \\
\hline AMI & 0.24 & 0.27 & 0.03 & 0.25 \\
\hline HF/CHF & 0.015 & 0.019 & 0.004 & 0.50 \\
\hline All deaths & 2.35 & 2.32 & -0.02 & 0.75 \\
\hline Female & & & & 0.03 \\
\hline All circ. & 1.19 & 1.07 & -0.12 & 0.03 \\
\hline IHD & 0.77 & 0.72 & -0.04 & 0.35 \\
\hline AMI & 0.23 & 0.24 & 0.01 & 0.75 \\
\hline HF/CHF & 0.026 & 0.020 & -0.01 & 0.37 \\
\hline All deaths & 2.29 & 2.05 & -0.24 & 0.003 \\
\hline
\end{tabular}

Abbreviations: AMI, acute myocardial infarction; ANOVA, analysis of variance; Circ., diseases of the circulatory system; $\mathrm{HF} / \mathrm{CHF}$, heart failure or congestive heart failure; IHD, ischemic heart disease.

${ }^{a}$ The analysis group thus includes the day of the Super Bowl (January 22, 1984), and the 14 days after the Super Bowl (January 22, 1984-February $5,1984,15$ days total).

${ }^{b}$ To remove the impact of the known peak in cardiac death rates around the holidays, the analysis excludes data from January 1 to 14 (14 days starting with New Year's Day).

${ }^{C}$ Rates expressed per day per 100000 population (using total deaths and average population).

${ }^{d}$ Estimated from a 1-way ANOVA with Super Bowl Day as the only factor.

${ }^{e}$ For the Super Bowl effect.

Data for those aged $<65$ and $\geq 65$ years are shown in Table 6 . Those aged $\geq 65$ had a small but significant reduction in all-cause mortality associated with the Super Bowl win.

Table 6. Analysis of Age and Deaths From Cardiac Causes for the 1984 Super Bowl and Subsequent 14 Days $^{a}$ vs All Other Days in January ${ }^{b}$ and February Between January 15, 1984, and February 1988

\begin{tabular}{|ccccc|}
\hline Cause of Death & Control $^{c}$ & $\begin{array}{c}\text { Super Bowl } \\
1980^{c}\end{array}$ & $\beta$ Estimate $^{d}$ & $P$ Value $^{e}$ \\
\hline Age $<65$ y & & & & \\
\hline All circ. & 0.22 & 0.23 & 0.01 & 0.51 \\
\hline IHD & 0.14 & 0.15 & 0.02 & 0.20 \\
\hline AMI & 0.05 & 0.05 & 0.005 & 0.49 \\
\hline HF/CHF & 0.003 & 0.0009 & -0.002 & 0.22 \\
\hline All deaths & 0.81 & 0.79 & -0.02 & 0.60 \\
\hline Age $\geq 65$ y & & 8.91 & -0.49 & 0.19 \\
\hline All circ. & 9.39 & 6.18 & -0.21 & 0.48 \\
\hline IHD & 6.39 & & & \\
\hline
\end{tabular}

Table 6. Continued

\begin{tabular}{|ccccc|}
\hline Cause of Death & Control $^{c}$ & $\begin{array}{c}\text { Super Bowl } \\
1980^{c}\end{array}$ & $\beta$ Estimate $^{d}$ & $P$ Value $^{e}$ \\
\hline AMI & 1.98 & 2.10 & 0.12 & 0.41 \\
\hline HF/CHF & 0.184 & 0.188 & 0.003 & 0.94 \\
\hline All deaths & 16.3 & 15.1 & -1.19 & 0.03 \\
\hline
\end{tabular}

Abbreviations: AMI, acute myocardial infarction; ANOVA, analysis of variance; Circ., diseases of the circulatory system; $\mathrm{HF} / \mathrm{CHF}$, heart failure or congestive heart failure; IHD, ischemic heart disease.

${ }^{a}$ The analysis group thus includes the day of the Super Bowl January 22, 1984), and the 14 days after the Super Bowl (January 22, 1984-February $5,1984,15$ days total).

${ }^{b}$ To remove the impact of the known peak in cardiac death rates around the holidays, the analysis excludes data from January 1 to 14 (14 days starting with New Year's Day).

${ }^{C}$ Rates expressed per day per 100000 population (using total deaths and average population).

${ }^{d}$ Estimated from a 1-way ANOVA with Super Bowl Day as the only factor.

${ }^{e}$ For the Super Bowl effect.

Data for white/Hispanic and nonwhite/non-Hispanic populations are shown in Table 7 . There were nonsignificant trends for reduction in all-cause mortality associated with

Table 7. Analysis of Race and Deaths From Cardiac Causes for the 1984 Super Bowl and Subsequent 14 Days $^{a}$ vs All Other Days in January ${ }^{b}$ and February Between January 15, 1984, and February 1988

\begin{tabular}{|lcccc|}
\hline Cause of Death & Control & $\begin{array}{c}\text { Super Bowl } \\
1984^{c}\end{array}$ & $\beta$ Estimate $^{d}$ & $P$ Value \\
\hline White/Hispanic & & & & \\
\hline All circ. & 1.19 & 1.16 & -0.03 & 0.47 \\
\hline IHD & 0.81 & 0.82 & 0.007 & 0.84 \\
\hline AMI & 0.25 & 0.27 & 0.02 & 0.35 \\
\hline HF/CHF & 0.022 & 0.021 & -0.0008 & 0.88 \\
\hline All deaths & 2.42 & 2.30 & -0.12 & 0.07 \\
\hline Nonwhite/non-Hispanic & & & & 0.0 .17 \\
\hline All circ. & 0.81 & 0.74 & -0.07 & 0.22 \\
\hline IHD & 0.49 & 0.41 & -0.07 & 0.11 \\
\hline AMI & 0.17 & 0.18 & 0.01 & 0.63 \\
\hline HF/CHF & 0.017 & 0.013 & -0.004 & 0.62 \\
\hline
\end{tabular}

Abbreviations: AMI, acute myocardial infarction; ANOVA, analysis of variance; Circ., diseases of the circulatory system; HF/CHF, heart failure or congestive heart failure; IHD, ischemic heart disease.

${ }^{a}$ The analysis group thus includes the day of the Super Bowl (January 22, 1984), and the 14 days after the Super Bowl (January 22, 1984-February $5,1984,15$ days total).

${ }^{b}$ To remove the impact of the known peak in cardiac death rates around the holidays, the analysis excludes data from January 1 to 14 (14 days starting with New Year's Day).

${ }^{C}$ Rates expressed per day per 100000 population (using total deaths and average population).

${ }^{d}$ Estimated from a 1-way ANOVA with Super Bowl Day as the only factor.

${ }^{e}$ For the Super Bowl effect. 
Table 8. $P$ Values From Linear Regression for Mortality Rate for the 1984 Super Bowl ${ }^{a}$

\begin{tabular}{|c|c|c|c|c|c|c|c|}
\hline Outcome & 1984 SB & Race & Sex & Age & SB Race & SB Sex & SB Age \\
\hline All-cause mortality & 0.07 & 0.0006 & $<0.0001$ & $<0.0001$ & 0.82 & 0.07 & 0.08 \\
\hline All heart deaths & 0.44 & 0.0003 & 0.0001 & $<0.0001$ & 0.94 & 0.12 & 0.44 \\
\hline IHD & 0.32 & $<0.0001$ & 0.004 & $<0.0001$ & 0.44 & 0.77 & 0.32 \\
\hline AMI & 0.38 & 0.06 & 0.09 & $<0.0001$ & 0.95 & 0.44 & 0.40 \\
\hline $\mathrm{HF} / \mathrm{CHF}$ & 0.73 & 0.96 & 0.43 & $<0.0001$ & 0.81 & 0.24 & 0.65 \\
\hline
\end{tabular}

the 1984 Super Bowl in both groups $(P=0.07$ for white/Hispanic; $P=0.08$ for nonwhite/non-Hispanic).

Table 8 shows $P$ values from linear regression analyses for death rate with the following variables included in the model: Super Bowl days, race, sex, age, and interactions for each of these covariates with the Super Bowl variable. There was a nonsignificant trend for interaction for allcause mortality between Super Bowl and sex $(P=0.07)$ and between Super Bowl and age $(P=0.08)$. The analyses use data from January 15 to February, 1984-1988. There are 227 days included in this interval, and there are 8 combinations of the 3 covariates $(2 \times$ race, $2 \times$ sex, $2 \times$ age), for a total of 1816 observations (independent death rates).

\section{Discussion}

It is known that stressors such as intense sporting events may increase cardiac event rates in fans, but there has been little data available on the demographics of these fans. ${ }^{8-11}$ Based on our linear regression analysis, our study suggested that Los Angeles' 1980 Super Bowl loss increased total and cardiac deaths in both men and women and triggered more deaths in older patients compared with younger patients. Conversely, the 1984 Super Bowl win showed a trend for reduction of death rates, slightly better in older than younger patients and in women more than men. There were no significant interactions between Super Bowl and race in our linear regression analyses.

The concept that sporting events may be associated with an increase in cardiac events was best studied in the European soccer literature. During the 2006 World Cup, when the German team played, there were increases in acute coronary syndromes and arrhythmias. ${ }^{8}$ The increase in cardiac events was more prominent in men than in women. Other reports of an increase in cardiac events and deaths suggest a prevalence in males. However, in our study there was no interaction between the 1980 Super Bowl loss and sex. In other words, there was an increase in total mortality as well as cardiac mortality in both men and women. To the best of our knowledge, this is the first report of an increase in cardiac events in women associated with the Super Bowl. Although the exact mechanism for this observation is not known, there are several possibilities. The Super Bowl may elicit an emotional response that is similar in US females and males, or perhaps a male's reaction to the Super Bowl loss adversely affected the emotional state of a female partner.

Older patients are of course more likely to have advanced coronary artery disease, but there is little data in the literature regarding triggering of cardiac events in older vs younger individuals. Whereas both older and younger people demonstrated an increase in total and cardiac mortality, the absolute increase in death rates was greater in older (2.6 per day) than in younger individuals $(0.11$ per day), and the regression model demonstrated a significant interaction between the Super Bowl variable, age, and all-cause mortality $(P<0.0001)$ as well as circulatory death $(P=0.0002)$. The mechanism for this finding is not known, but it may be that atherosclerotic plaques in elderly patients are more vulnerable to rupture in response to an increase in catecholamines or stimulation of the sympathetic nervous system than are plaques in younger patients.

Unlike the 1980 Super Bowl loss, the 1984 Super Bowl win, just a few years later and on a similar date (January 22 , 1984, vs January 20, 1980), was not associated with an increase in total mortality or cardiac mortality. In fact, there was a slight reduction in mortality. ${ }^{9}$ This signal tended to be greatest in women and older individuals. As described in the Background section of this article, there were marked differences in the nature of these 2 Super Bowl games, beyond the fact that the 1980 game was a loss for Los Angeles and the 1984 game was a win. The 1980 loss was a much more intense game, with frequent lead changes. Fan loyalty may also have been greater in 1980 than in 1984, because the losing Rams had been in Los Angeles since 1946, whereas in 1984 the winning Raiders had only been playing in Los Angeles for a few years. In addition, the 1980 Super Bowl loss happened locally, in Pasadena, and the 1984 Super Bowl win took place out of state, in Florida. Hence, the emotional involvement of fans was likely greater for the 1980 Super Bowl loss.

\section{Conclusion}

Whether a major sporting event (win or loss) can trigger death, including cardiac death, may in part be dependent upon demographics. The 1980 Super Bowl loss triggered more deaths in older patients compared with younger patients. It did trigger deaths in both men and women. In addition, there was a nonsignificant trend for a Super 
Bowl win to reduce death slightly better in older than in younger patients, and in women.

\section{References}

1. Ridker PM, Libby P. Risk factors for atherothrombotic disease. In: Libby P, Bonow RO, Mann DL, et al, eds. Braunwald's Heart Disease: A Textbook of Cardiovascular Medicine. 8th ed. Philadelphia, PA: Saunders; 2007: chapter 39. Pages 1003-1026.

2. Tofler GH, Stone PH, Maclure M, et al. Analysis of possible triggers of acute myocardial infarction (the MILIS study). Am J Cardiol. 1990;66:22-27.

3. Muller JE, Tofler GH, Stone PH. Circadian variation and triggers of onset of acute cardiovascular disease. Circulation. 1989;79. 733-743.

4. Kloner RA. Natural and unnatural triggers of myocardial infarction. Prog Cardiovasc Dis. 2006;48:285-300.

5. Muller JE, Abela GS, Nesto RW, et al. Triggers, acute risk factors and vulnerable plaques: the lexicon of a new frontier. J Am Coll Cardiol. 1994;23:809-813.
6. Mittleman MA, Maclure M, Tofler GH, et al; Determinants of Myocardial Infarction Onset Study Investigators. Triggering of acute myocardial infarction by heavy physical exertion. Protection against triggering by regular exertion. N Engl J Med. 1993;329: 1677-1683

7. Muller JE, Tofler GH. Triggering and hourly variation of onset of arterial thrombosis. Ann Epidemiol. 1992;2:393-405.

8. Wilbert-Lampen V, Leistner D, Greven S, et al. Cardiovascular events during World Cup soccer. N Engl J Med. 2008;358: 475-483

9. Kloner RA, McDonald S, Leeka J, et al. Comparison of total and cardiovascular death rates in the same city during a losing versus winning Super Bowl championship. Am J Cardiol. 2009;103: 1647-1650.

10. Witte DR, Bots ML, Hoes AW, et al. Cardiovascular mortality in Dutch men during 1996 European football championship: longitudinal population study. BMJ. 2000;321:1552-1554.

11. Carroll D, Ebrahim S, Tilling K, et al. Admissions for myocardial infarction and World Cup football: database survey. BMJ. 2002;325: 1439-1442. 